\title{
A RADON-NIKODYM TYPE THEOREM FOR $\alpha$-COMPLETELY POSITIVE MAPS ON GROUPS
}

\author{
MARIA JOIŢA
}

Abstract. We show that an operator valued $\alpha$-completely positive map on a group $G$ is given by a unitary representation of $G$ on a Krein space which satisfies certain conditions. Moreover, two such of unitary representations, which are unitarily equivalent, define the same $\alpha$-completely positive map. Also we introduce a pre-order relation on the collection of $\alpha$-completely positive maps on a group and we characterize this relation in terms of the unitary representation associated to each map.

Mathematics subject classification (2010): Primary 43A35, 46C50, 46L05.

Keywords and phrases: $\alpha$-completely positive map on groups, Radon-Nikodym theorem, unitary representation on Krein spaces.

\section{REFERENCES}

[1] J. P. Antoine, S. ÔTA, Unbounded GNS representations of $*$-algebra in a Krein space, Lett. Math. Phys. 18 (1989), 4, 267-274.

[2] J. HEO, $\alpha$-completely positive maps of group systems and Krein module representations, J. Math. Analysis Appl. 409 (2014), 544-555.

[3] J. HEO, U. C. JI, Radon-Nikodým type theorem for $\alpha$-completely positive maps, J. Math. Phys. 51, 103505, 2010.

[4] J. Heo, J. P. Hong, U. C. Ji, On KSGNS representations on Krein $C^{*}$-modules, J. Math. Phys. 51, $053504,2010$.

[5] G. Hofmann, An explicit realization of a GNS representation in a Krein-space, Publ. Res. Inst. Math. Sci. 29 (1993), 2, 267-287.

[6] L. JАКОВCZYK, F. STROCCHI, Euclidean formulation of quantum field theory without positivity, Commun. Math. Phys. 119 (1988), no. 4, 529-541.

[7] K. R. PARTHASARATHY, Comparison of completely positive maps on a $C^{*}$-algebra and a Lebesgue decomposition theorem, in Athens Conference on Applied Probability and Time Series Analysis I, Lecture Notes in Statistics Vol. 114, Springer-Verlag, New York, 1996, pp. 34-54.

[8] M. RAGINSKY, Radon-Nikodým derivatives of quantum operations, J. Math. Phys. 44, 5003, 2003. 